\title{
Clocking in the face of unpredictability beyond quantum uncertainty
}

\author{
F. Hadi Madjid ${ }^{a}$ and John M. Myers ${ }^{b}$ \\ ${ }^{a} 82$ Powers Road, Concord, MA 01742, USA; \\ ${ }^{b}$ Harvard School of Engineering and Applied Sciences, Cambridge, MA 02138, \\ USA
}

\begin{abstract}
In earlier papers we showed unpredictability beyond quantum uncertainty in atomic clocks, ensuing from a proven gap between given evidence and explanations of that evidence. Here we reconceive a clock, not as an isolated entity, but as enmeshed in a self-adjusting communications network adapted to one or another particular investigation, in contact with an unpredictable environment. From the practical uses of clocks, we abstract a clock enlivened with the computational capacity of a Turing machine, modified to transmit and to receive numerical communications. Such "live clocks" phase the steps of their computations to mesh with the arrival of transmitted numbers. We lift this phasing, known in digital communications, to a principle of logical synchronization, distinct from the synchronization defined by Einstein in special relativity.

Logical synchronization elevates digital communication to a topic in physics, including applications to biology. One explores how feedback loops in clocking affect numerical signaling among entities functioning in the face of unpredictable influences, making the influences themselves into subjects of investigation. The formulation of communications networks in terms of live clocks extends information theory by expressing the need to actively maintain communications channels, and potentially, to create or drop them.

We show how networks of live clocks are presupposed by the concept of coordinates in a spacetime. A network serves as an organizing principle, even when the concept of the rigid body that anchors a special-relativistic coordinate system is inapplicable, as is the case, for example, in a generic curved spacetime.
\end{abstract}

\section{INTRODUCTION}

Discoveries in physics involve unpredicted events, whether of the imagination of theorists or the surprises of unpredicted experimental evidence. Still, one can wonder: if scientists were smarter, could they have predicted the events that have come as surprises? Within the framework of quantum theory, the answer is "not on the basis of experimental evidence." As is explained in Sec. 2, quantum

Further author information: (Send correspondence to J.M.M.)

J.M.M.: E-mail: myers@seas.harvard.edu, Telephone: 16174955263

F.H.M.: E-mail: gmadjid@aol.com, Telephone: 19783691808 
explanations depend on guesses that are subject to revision in the face of future evidence, not only as a practical matter but as a matter of principle, demonstrating an inescapable unpredictability beyond the probabilistic uncertainty of quantum mechanics.

The proven dependence of explanations on guesswork promotes the aspiration, never quite attainable, to separate explanations from evidence, and to attend to the sources of both. I find a path to discovery when I recognize that my explanation cannot be the only one possible. This recognition of a certain freedom in choosing explanations may be important to philosophy, but does it matter to physics? To begin with, because investigations draw on surprises, both from external outcomes and from acts of our own guessing and making of assumptions, we who investigate are not interchangeable: You experience what I do not, and I can know what you find out only to the extent of our communication. Today investigators are often linked to sensors, to actuators, and to each other by a computer-mediated communications network. Experimental activity, including encounters with unpredictability, is reflected in such a network, both in the records held in the computers of the network and in the timing of communications within the network. Networks involve feedback. Indeed, lots of biological and engineered mechanisms involve networks that respond to unpredicted deviations from some aiming point. We want to lift the feedback that takes place in a computer-mediated system from the province of engineering to a form suitable for application in theoretical physics.

As an application of feedback in computer-mediated networks, we consider investigations of motion. The motion of an object can be thought of or measured only in relation to organized motions of other objects that serve as a reference background against which to see the motion of the object under investigation. Here we argue that recognizing the gap between evidence and its explanations forces a reconception of reference motions, away from the a rigid system of equations and measurement units, conceptually tied to a single investigator, to a pattern of motion maintained by investigators and their devices, linked in some chosen communications network that the investigators create and maintain, operating in an environment of surprise and guesswork.

To develop the concept of a networked reference for motion we start by retracing the topic, opened up a century ago by Einstein, of spatially and temporally locating events of interest. At root, this is a problem for every person from infancy on. Right now I feel my fingers on the keyboard, the reach of my arm for the coffee cup, the chair I sit in; I see the walls of the office and, through the window, the bare trees and the snow lingering in this late-coming season of spring. Or think of Galileo comparing the motion of a rolling ball against himself, e.g. his own heart beat; then as science progresses he (and we who follow after him) delegate the beats of our reference to a more stable external clock. A clock and a flat floor make a background for motion; however, reading a distant clock is an issue.

The prevalent way to think of a background against which to define motion is a coordinate system, but the abstraction that generates a coordinate system obscures the reference motions that necessarily compose such a background. To recover the reference motions intrinsic to a coordinate system, we review a little history. Today's notion of a coordinate system stems from the theories of special and general relativity. Commenting on how he developed those theories, Einstein spoke of the need to "drag down from the Olympian fields of Plato the fundamental ideas of thought in natural science, and to attempt to reveal their earthly lineage ..." 1 The earthly lineage leading to the theory of special relativity lies in the railroad age of the 1800's, in which the development of telegraphy provided the means to synchronize clocks to tell "time" spread across the span of telegraphy. Einstein abstracted 
the practice of synchronizing clocks by the exchange of telegraph signals into the theory of special relativity. Building on his bold hypothesis about light speed, he defined the synchronization of imagined proper clocks in terms of clock readings at the transmission and reception of idealized light signals. He defined a coordinate frame relative to an imagined rigid body in terms of signals exchanged among synchronized proper clocks fixed to the body. This definition tied the concept of a rigid body to a pattern of signal exchange among proper clocks, thereby relativizing the notion of rigid. Without clocks and the motion of the signals by which their synchronization is defined, there can be no concept of a Lorentz coordinate system.

In general relativity, no extended body can be rigid, but the equations that define a metric tensor field are still imagined as fixed. For astronomy and geodesy, the International Astronomical Union (IAU) employs a notion of a 'reference system' incorporating fixed equations defining a metric tensor field. ${ }^{2}$ Although obtained by dragging down ideas "from the Olympian fields of Plato," Einstein's synchronization criterion for special relativity, as well as the general-relativistic reference systems, are elevated to a blackboard of Plato, suitable for armchair contemplation, but remote from any active experimental setting. From the perspective of a physics that faces the unpredictable, the blackboard on which equations are written needs to be split up and made dynamic, with the equations of a reference system copied into the separate memories of the computers of the network that links the investigators and their automated devices. Copied into these memories, the equations become available to distributed real-time computations essential to the feedback that maintains the network; furthermore, on occasion, the equations written in one or another computer memory can be modified in response to unpredictable happenings.

What can be investigated depends in part on how the computers of a network can communicate. In Sec. 3 we represent a real-time process-control computer that takes part in a network as a modified Turing machine stepped by a clock, and we explore the form of synchronization, distinct from Einstein's, necessary to the transmission of numbers from one Turing machine to another. To deal with communications between Turing machines, it will be necessary for the clock that steps a Turing machine to tick at a rate that can be adjusted by commands issuing from that machine. We call such an adjustable clock in combination with the Turing machine that regulates its rate a live clock. (Such rate adjustment is found in the cesium clocks that generate International Atomic Time (TAI) ${ }^{3}$ ) Because the evidence recorded by one live clock differs unpredictably from that recorded by another, unlike proper clocks postulated in relativity theory, each live clock is unique.

A live clock can receive a number from a detector, such as a photodetector, that acts from a computational point of view as an oracle, the workings of which are unknowable. ${ }^{4}$ Other oracle numbers show up as clock readings made by one live clock and transmitted to another live clock. Both human and automated communication of numbers involves cycling through phases. Think of the cycle of a bucket brigade, in which a person turns to receive a bucket just as a another person offers the bucket. This need for cycling through phases structures the communications in which live clocks participate, leading to a definition of the logical synchronization of a channel from one live clock to another, distinct from the synchronization defined by Einstein. The need for logical synchronization of its channels constrains the design of a network of live clocks.

To maintain logical synchronization of the channels of a network of live clocks requires feedback because, among other reasons, at the level of precision with which atomic clocks can be measured, 
"no two clocks tick alike.' ${ }^{3}$ In this feedback, the live clocks of a network steer their tick rates and accelerations in response to unpredictable measured deviations from an aiming point. The aiming point is chosen to express desired communications channels, and steering toward that aiming point is determined by control equations programmed into the live clocks.

Designing and operating the channels of a network and the corresponding control equations requires a hypothesis concerning how signals propagate from one live clock to another. It is a great convenience in making such a hypothesis to draw on the concept of a spacetime manifold; however, evidence in the form of clock readings is independent of any hypothesis about signal propagation, and therefore can be used to test a hypothesis, regardless of whether the hypothesis invokes the concept of a spacetime manifold. Thus there is great freedom in the choosing of hypotheses about signal propagation. In addition to serving as a background against which to think about and measure motion, a network of live clocks serves as a tool to investigate the propagation of the signals by which the live clocks of the network communicate.

Among candidate hypotheses about propagation, those based on a general-relativistic spacetime manifold commend themselves as an appropriate first case for theoretical purposes. It is especially interesting that a generic curved spacetime manifold rules out a fixed general-purpose pattern of signal exchange, and so calls for tailoring a network of live clocks to whatever situation is under investigation. In Sec. 4 we discuss a network of live clocks, designed assuming a spacetime manifold and intended to serve as an adaptive background for motion and as a tool to investigate gravitation. The operation of the network involves a cycle of provisionally assuming a metric tensor field, using it to construct an aiming point in terms of relations among clock readings toward which to steer the live clocks, implementing the corresponding control equations, and evaluating the resulting deviations from the aiming point. If the deviations cannot be held within tolerable bounds, the investigators, drawing on the measured clock readings, guess a different metric tensor field, and the cycle continues.

Sec. 5 offers a brief perspective on how recognizing essential unpredictability impacts the application of physical concepts, based on the dependence of both explanations and experiments on a dynamically evolving "tree of assumptions."

\section{UNPREDICTABILITY IMPLIED BY QUANTUM THEORY}

Given a quantum state and a measurement operator, quantum theory sets up predictions of a probability measure over a set of possible outcomes, and quantum uncertainty denotes a spread in the probability measure. Quantum theory, however, implies something beyond uncertainty, namely a kind of unpredictability, in some cases involving the introduction of possibilities previously unforeseen. In earlier work we proved that:

1. Infinitely many explanations are consistent with any given evidence. While each explanation fits the given evidence, the explanations have conflicting implications for evidence obtainable from experiments not yet performed. Once candidate explanations are proposed, one can bet on them-in effect, assigning rough probabilities to them. But in the face of an experimental surprise, before any explanation is proposed, no evidence can determine the probability to assign to any of the numberless not-yet-known explanations that fit given evidence. For this reason, 
explanations as hypotheses chosen for testing come as surprises, unpredictable even to the person who chooses them. $5 \cdot-7$

2. To choose one or a few explanations requires reaching beyond any predictive logic and beyond the guidance of probabilities to make a guess. ${ }^{6}$

3. Because it excludes conflicting explanations that fit the given evidence equally well, any guessed explanation is susceptible to refutation by surprising results coming from future experiments, prompting its replacement by some other explanation. $\frac{8}{8}$

4. Choosing explanations as hypotheses to be tested by experiments takes place in an open cycle, alternating between subjective surprises of guessing hypotheses and objective surprises of unexpected experimental results..$^{8}$ Thus not only do explanations depend on assumptions but so do experiments: their design and the interpretation of their results depend on explanations that rest on layers of guesswork.

5. In addition to the unpredictability of guesses that choose explanations, quantum uncertainty makes individual occurrences of outcomes of generic measurements unpredictable. Two situations are to be distinguished:

(a) An experimenter tests a predicted probability measure by repeating a measurement many times and comparing measured relative frequencies against predicted probabilities. In this situation individual occurrences of outcomes matter only as they enter tallies, ratios of which become relative frequencies.

(b) As in applications of quantum decision theory, ${ }^{910}$ an experimenter has a prior probability measure, sometimes only a guess, for the state being measured and uses a single unpredictable result or a short run of results to together with Bayes rule to decide on a parameter value that characterizes the state, acting promptly on that decision. (Such prompt action takes place in the feedback loops by which atomic clocks must be steered in frequency. ${ }^{3}$ )

Although surprises in physics are familiar as a practical matter, before the proof one might try to relegate them to the status of exceptions to the progress of science. The proof gives theoretical force to the unpredictability of surprises and guesses, so that the implications of this unpredictability can be explored. If a guess is necessary for an explanation, what does that mean for discovery? Who or what makes a guess? In particular, the dependence of an explanation of given evidence on guesswork motivates more attention to two things: (a) the distinction between explanations and the experiences that one explains, and (b) the entity, long neglected in physics, in which evidence, calculation, and guesswork meet.

\section{CLOCKS THAT COMPUTE AND COMMUNICATE}

When investigators and their devices are linked to one another by a computer-mediated network, the network serves as a tool for the investigation; it also houses the results of the investigation in records distributed in the memories of its computers. We model a computer in a network by a clock joined to a Turing machine that is modified to permit communication with other such machines and with an 
unpredictable environment containing sensors such a photodetectors. The clock has provision for its rate of ticking to be adjusted on the fly by commands from the Turing machine. We call such a clock together with the Turing machine a live clock. The live clock models an entity in which evidence, guesswork, and calculation meet. It is the key component in any of various communications networks against which to reference the motion of objects under investigation. Live clocks enable a networked reference for motion to adapt to the needs of particular investigations.

Turing represented mathematically the activity of calculating by what is now called a Turing machine, imagined as operating in a sequence of 'moments' interspersed by 'moves'. The Turing machine has a working memory in the form of a tape. At any moment the machine scans one square of the tape, on which it can read or write a single character of some alphabet that need not be more than the binary set $\{0,1\}$.11 A move as defined in the mathematics of Turing machines consists only of the logical relation between the machine at one moment and the machine at the next moment. Thus the Turing machine models the logic of computation by relating a program and a record in memory to an output in memory, without regard to timing. Two computations executing at different speeds can be represented in their logic by the same sequence of moments and moves. Because the Turing machine is indifferent to timing, it cannot calculate the tick that steps its moves, and so cannot by itself express the physical motion of computation in the way that the live clock does.

People or machines as calculators do lots of things, such as taking a break or fetching new supplies, that Turing abstracted out of sight. In so doing he sealed the Turing machine off from receiving or transmitting communications to anything outside its computational activity. He did, however, open the door to communication a crack by briefly discussing a variant that he called a choice machine which, on occasion, could wait for an external input. ${ }^{11}$ In adapting it as a component of a live clock, we modify the Turing machine further so that the live clock can not only receive characters of its alphabet from an external environment, but can also transmit characters to that environment. The environment can include other live clocks.

\subsection{Cycle of moves and moments of computation}

A live clock operates in a cycle of receiving unpredictable information from an environment, storing that information in memory, computing a response, and issuing that response to the environment. The cycle has subcycles, and at the finest level is composed of moments and moves of the clock-driven Turing machine that makes up the live clock. For a live clock to take part in communication, its moments and moves have to be regulated to avoid the logical conflict of a collision between writing into memory and reading from memory. (In human terms this is the collision between trying to speak and listen at the same time.) To avoid this conflict, the modified Turing machine is driven by the adjustable clock through a cycle with two phases of moves and two phases of moments, with reading from memory taking place in a phase separated from a phase of writing into memory.

A cycle of the live clock corresponds to a unit interval of the readings of its adjustable clock. A reading $t$ of a live clock can be expressed in the form $m . \phi_{m}$ where an integer $m$ indicates the count of cycles and $\phi_{m}$ is the phase within the cycle. We choose the convention that $-1 / 2<\phi_{m} \leq 1 / 2$. (It is not necessary to think of the signals as points in time; it suffices to think of a point reference within the signal.) 
Guesses, such as guessed explanations, enter the memory of a live clock as inputs from outside of the live clock, and are written into its memory during phases of writing. We give no further explanation of this "outside" from which guesses are assumed to come. Because live clocks have memories, they can record and make use of readings of other live clocks and indeed their own readings - a mechanical analog of "self-awareness."

\subsection{Logically synchronizing the communications between live clocks}

To express the clocking of actual or contemplated communications between one live clock and another, we follow Shannon in speaking of a communications channel; however we augment his informationtheoretic concept of a channel ${ }^{12}$ with the live-clock readings at the transmission and reception of character-bearing signals. ${ }^{3}$ Each character transmitted from a live clock $A$ to a live clock $B$ is associated with a reading of live clock $A$ of the form $m . \phi_{m}$ at the transmission and with a reading of live clock $B$ of the form $n . \phi_{n}$ at the reception. A channel from $A$ to $B$ includes a set of such pairs of readings of the transmitting and the receiving live clocks. The necessity of avoiding a conflict between reading and writing imposes a constraint on the phases of reception.

Proposition: A character can propagate from one live clock to another only if the character arrives within the writing phase of the receiving live clock.

When this phase constraint is met for a channel between a transmitting live clock and a receiving live clock, we say the receiving live clock is logically synchronized to the transmitting live clock. Logical synchronization is analogous to the coordination between neighboring people in a bucket brigade, or that between players tossing a ball back and forth, where the arrival of the ball must be within a player's 'phase of catching'. In this way the notion of a channel is expanded to include the clock readings that indicate phases of signal arrivals that have to be controlled in order for the logical synchronization of the channel to be maintained. (While in many cases the integers in clock readings that count cycles can be definitely specified, the phases are never exactly predictable.) We model the phase of writing at which a live clock can receive a character as corresponding to

$$
|\phi|<(1-\eta) / 2
$$

where $\eta$ (with $0<\eta<1$ ) is a phase interval that makes room for reading.

Logically synchronizing a channel means bringing about the condition (1) on phases at which signals arrive. Once logical synchronization is acquired, maintaining it typically requires more or less continually adjusting the rates of ticking and the acceleration of one or both of the live clocks, in order to steer the phases of arriving characters toward a suitable aiming point, say some $\phi_{0}$. In the simplest case, this aiming point $\phi_{0}$ is 0 . When a live clock receives signals over more than one channel, it measures its own clock readings at all the receptions and takes all the phase deviations into account to steer its own tick rate and acceleration. The clock readings made for the purpose of maintaining logical synchronization are not interpreted as indicating "the time"; instead, like the readings of a clock that is being adjusted by a clockmaker, they indicate the amount by which the live clock needs to be adjusted.

Relations among readings of live clocks that contribute to an aiming point for a network include what we call echo counts, closely related to distances defined by radar: 
Definition of echo count: Suppose that at its reading $m .0$ a live clock $A$ transmits a signal at to a live clock $B$, and the first signal that $B$ can transmit back to $A$ after receiving $A$ 's signal reaches $A$ at $m^{\prime} . \phi^{\prime}$; then the quantity $m^{\prime} . \phi^{\prime}-m .0$ will be called the echo count $\Delta_{A B A}$ at $m$.

The need for logical synchronization of the channels strongly constrains the design of a network of live clocks. In Sec. 4 we will see how the ticks of the clocks are allowable only within intersections of "stripes in spacetime". [-

Steering toward an aiming point involving echo counts depends on one live clock receiving signals that convey readings of other live clocks. The possibility of wireless communication requires that such a signal from a live clock $A$ to a live clock $B$ carry an identifier of $A$, so that the receiver $B$ can tell the source of the signal. With these identifiers, the channels of a network of live clocks correspond to directed edges of a graph, leading to a data structure for readings of live clocks of a network illustrated in [3] and discussed at more length in terms of marked graphs and Petri nets in [13]. Such data structures for a network can reside in the memory of a live clock, so the live clock has a picture of the network in which it participates.

\section{NUMERICAL COMMUNICATION AS A FRAME OF REFERENCE}

With unpredictability established in Sec. 2 and the consequent need for feedback to support logical synchronization established in Sec. 3, we now show how networks of live clocks can offer reference patterns of motion against which to think about and measure the motion of some object of interest. The following subsections tell:

1. how the concept of a coordinate system as the prevalent reference for motion depends on Einstein's synchronization criterion and thereby implicitly depends on a network of live clocks;

2. how, assuming a flat spacetime, the requirement for logical synchronization constrains a network of live clocks that approximates a Lorentz frame;

3. how logical synchronization can be maintained in cases that preclude Einstein synchronization;

4. how, in the theory of curved spacetime, there can be no large rigid body and no all-purpose network of live clocks to realize a background to motion;

5. how a network of live clocks serves as a tool to discover features of its unpredictable environment, including features of gravitation;

6. how predictability meets unpredictabilty on a Turing tape. 


\subsection{Coordinate frames presuppose communication between 'self-aware' clocks}

To see that a coordinate system presupposes a network of live clocks, recall Einstein's definition of synchronization in special relativity. Einstein conceived of the location of an arbitrary event as a clock tick coincident with the event. This makes a spacetime of possible locations of events into a potential for clock ticks, so that for any event one can think of a tick coincident with it. But the tick is significant only in relation to other ticks. By imagining a system of light signals propagating between imagined proper clocks, Einstein defined the synchronization of proper clocks fixed to a non-rotating, rigid body in free fall (i.e., a Lorentz frame) and co-defined "time" as the readings of such proper clocks, with the implications that distance from proper clock $A$ to proper clock $B$ is defined, as in radar, in terms of the duration at $A$ from the transmission of a light signal to the return of its echo from $B$. (If we imagine proper clocks as live clocks, this difference in readings of clock $A$ is an echo count.) Specifically, according to Einstein's definition of the synchronization of proper clocks fixed to a Lorentz frame, $\frac{14}{, 14}$ clock $B$ is synchronous to clock $A$ if at any $A$-reading $t_{A}, A$ could send a signal reaching $B$ at $B$ reading $t_{B}$, such that an echo from $B$ would reach $A$ at $A$-reading $t_{A}^{\prime}$, satisfying the criterion

$$
t_{B}=\frac{1}{2}\left(t_{A}+t_{A}^{\prime}\right) .
$$

A Lorentz coordinate system presupposes Einstein's synchronization and thereby presupposes the possibility of proper clocks that exchange light signals - an abstraction of a grid-like network of live clocks and their signals, in which every pair of live clocks exhibits constant echo counts and a constant Einstein synchronization relation with its neighbors.

The clock readings along the history of a moving object constitute a description of its motion, but how does that description become knowable to the investigators? The concept of a Lorentz coordinate system obscures the information processing needed for clock readings to become known to investigators. In contrast, live clocks allow for the local recording of their readings and the subsequent transmission of readings from one live clock to another. They also give expression to the necessity, stemming from quantum theory, to respond to unpredictable deviations from desired relations.

Although special relativity imagines proper clocks free of drift, quantum theory asserts an irreducible drift in tick rates and echo counts, ${ }^{\sqrt{3}}$ so that the readings of nearby, unadjusted clocks wander apart without bound unless their ticking is adjusted. When we recognize the inescapability of this drift, the Einstein synchronization relations (2) and the echo counts can no longer be taken as externally supplied facts, but instead work as aiming points chosen by investigators, toward which to steer the operation of a network of live clocks. This steering, if it can be accomplished, must depend on feedback of unpredictable measured deviations from the aiming point into the steering of the tick rates and the accelerations of the clocks of a network.

To be steerable on the basis of measured deviations, the clocks must act as live clocks. Furthermore, feedback that steers toward Einstein's synchronization requires the communication of numerals from one live clock to another. For example, consider a network of live clocks, subject to unpredictable drift, in which each live clock aims to satisfy an aiming point expressed as a set of relations among readings of itself and neighboring live clocks. Suppose the aiming point is comprised of the Eq. (2) along with constant echo counts for selected channels to nearby live clocks. Because of drift, measured clock readings deviate from the relations that constitute the aiming point. In response to the deviations from the aiming point, each live clock promptly adjusts its tick rate and acceleration. A live clock cannot 
wait for an external intelligence to compute the deviations exhibited by measured, unpredictable clock readings; it must itself compute the relevant deviations and its own response to these. For example, a clock $B$ computes a deviation that involves the difference between its own reading at its reception of a signal from a nearby clock $A$ and the reading of $A$ at the transmission of that signal. Thus $B$ can know how it deviates from being Einstein-synchronous with $A$ only if readings of $A$ 's clock are communicated to $B$. It is not enough for a light signal from $A$ to reach $B$; in addition the light signal has to convey the numerical reading of $A$ at the transmission of that signal. Hence steering toward Einstein's synchronization criterion of Eq. (2) requires the communication of numbers, which requires logical synchronization.

\subsection{Constraints of logical synchronization limit realizations of coordinates}

The requirement for logical synchronization constrains the arrangements of clocks that can satisfy Einstein's synchronization criterion. To show this, it is instructive to consider the case of ideal logical synchronization in which all phases at signal receptions are zero. Take the theoretical case of 8 live clocks located at the corners of a cube in a flat or conformally flat spacetime. Let each edge of the cube correspond to logically synchronized channels in both directions, all with zero phases. In that case channels across the face diagonals cannot have zero phases, simply because the ratio of the length of a face diagonal to an edge is irrational. But if the cube is replaced by a rectangular brick, the brick can be chosen as an Euler brick for which edges and face diagonals can both have integer lengths, 15 allowing a pattern of signaling having receptive phases for signals along edges of the brick and along its face diagonals to all be zero. In effect the requirement for logical synchronization puts stripes on spacetime for allowable configurations networks of live clocks. ${ }^{[3}$ Nonetheless, within any theory that assumes a flat or conformally flat spacetime, a three dimensional grid of live clocks exchanging signals along edges and face diagonals of Euler bricks serves as a universally applicable pattern of motion for a coordinate system. We will soon see a difference in this regard between a conformally flat spacetime and a generic curved spacetime.

\subsection{Logical synchronization where Einstein synchronization fails}

While realizing Einstein's synchronization requires logical synchronization among pairs of live clocks, the converse does not hold: cases of logically synchronized networks can be demonstrated for which Einstein synchronization is impossible. These cases include live clocks in relative motion, subject to the Doppler effect, live clocks on a rotating platform, subject to the Sagnac effect, and live clocks in the presence of gravitational fields. For this and other reasons it becomes an interesting scientific topic to explore possible patterns of signal exchange among logically synchronized live clocks. The topic includes the application of logical synchronization to the study of gravitation.

\subsection{Live clocks under the assumption of spacetime curvature}

Bringing hypotheses of one or another curved metric tensor field into consideration reveals a conceptual challenge to the definition and measurement of motion. As amply confirmed by experiments with space vehicles, by astronomical observations, and by experience with the Global Positioning System (GPS), the theory of general relativity asserts that the flat metric tensor field of special relativity and its concomitant realization by a pattern of light signals between clocks can only be approximated over 
a region the size of which must decrease as the instability of clocks decreases. If generic curvature is significant, there can be no arbitrarily fine, three-dimensional grid of signal-exchanging live clocks that pairwise satisfy Eq. (2), so that the universally applicable pattern of motion for a coordinate sys-

tem noted for flat spacetime is ruled out. ${ }^{16}$ This effect of generic spacetime curvature is a consequence of general relativity alone without invoking logical synchronization. Because the concept of a rigid body hinges on maintaining Einstein synchronization among the proper clocks thought of as elements of that body, it is apparent that the theory of general relativity is incompatible with any exactly rigid body.

\subsubsection{How to accept "no rigid bodies"}

A pattern of motion taken as a tool for determining my own motion serves as a tool for navigation, perhaps best appreciated in circumstances of its absence, as when, perhaps on a hike, I am lost. The thought of being lost is scary. It is soothing to imagine a flat coordinate system, relative to which the distant and the immediate are brought into relation, the ground under my feet stays put, and navigation would be straightforward. But if the earth shifts, as at some level of precision it always does, how is one to think? And at levels of precision made possible by modern clocks, there can be no rigid body on which to stand, nor can there be a fixed background of communication. It becomes necessary to search for a background pattern and to adjust it if increased demands for precision or unforeseen circumstances make the sought pattern unrealizable. If needs to adjust are in the cards, it is perhaps better to be nimble. Recognizing unpredictability can be a first step toward that nimbleness.

\subsection{Logical synchronization opens up possibilities for discovery}

While we have spoken of spacetime coordinates, the concept of a network of live clocks as a reference for motion makes no assumption of a spacetime manifold or of a coordinate system. To design an aiming point of desired relations among clock readings for a network of live clocks, however, one needs to invoke some hypothesis about the propagation of signals from one live clock to another live clock. What makes a workable hypothesis depends on circumstances, for example whether the signal is conveyed by light in vacuum, by light in some medium such as optical fiber, or by nerve pulses, etc.

As a first specific case appropriate to theoretical physics, consider live clocks on space vehicles linked in a communications network for an experiment aimed at discovering features of gravitation that affect the network. Suppose the experiment employs a theory of of signals as light-like geodesics in some specified spacetime manifold. Then the theory of propagations of signals among live clocks depends on the curvature of a metric tensor field hypothesized for the spacetime manifold. To maintain logical synchronization, the live clocks employ control equations that depend not only on the investigators' basic assumption of a (possibly curved) spacetime manifold, but also on an additional provisional hypothesis of a particular metric tensor field, from which they arrive at a model of signal propagation, necessary to designing an aiming point specified in terms of relations among readings of live clocks at the transmission and the reception of light signals. The control equations depend on the provisional metric tensor field, and, together with that metric tensor field, are subject to revision when difficulties in maintaining logical synchronization are encountered.

For example, suppose the investigators guess that their space vehicles behave as if they were in a region of spacetime that has a metric that is flat to within the tolerances they can achieve with their 
live clocks. To test this guess, the investigators choose relations among clock readings as an aiming point under the provisional hypothesis of a flat spacetime. If, counter to their guess, the live clocks deviate too much from the aiming point, that deviation indicates the effect of a Weyl curvature of the spacetime.

In [3] a specific case of a cluster of 5 space vehicles, each equipped with a live clock, is analyzed for its capacity to measure unpredictable changes in gravitation by utilizing feedback to adjust clock rates and clock accelerations to generate a background motion maintained by computer-mediated feedback in response to unpredictable events. Gravitational effects are then detectable as they influence the channels found to be implementable. The investigation of gravitational effects thus involves a cycle of hypothesizing a metric tensor field and testing the implications of that hypothesis for clock readings against those recorded by the 5 live clocks.

Under hypotheses defining the second as a measuring unit in the International System of Units (SI), ${ }^{3}$ and with sufficiently stable live clocks, such a spaceborne network can potentially measure ripples in gravitation, something already pursued by other but related means by the Laser Interferometer Gravitational Wave Observatory (LIGO) ${ }^{17}$ We suspect that LIGO in fact illustrates how the use of feedback that responds to unpredictable events allows a precision in the background of measurement otherwise unattainable, and hence opens experimental inquiry into effects otherwise invisible. The complexity of LIGO, however, led us to point to the above arrangement of devices that, although likely difficult to implement, is conceptually simpler in its use of feedback that responds to the unpredictable.

\subsection{Predictability meets unpredictabilty on a Turing tape}

As represented in the memory of a live clock a variable can be thought of as a stretch of a Turing tape. A live clock engaged in a feedback loop houses separate variables-separate stretches of its Turing tape - for an aiming point derived from a hypothesis as distinct from a measured, unpredictable deviation from that aiming point. While both variables for aiming points and variables for deviations can experience changes in their values, typically the rate of change is relatively high for a deviation and low for an aiming point.

Important to logical synchronizaton is the distinction between a count of cycles recorded on tape at the receipt of a signal over a logically synchronized channel and the recorded phase of the cycle at which the signal arrives. The count is so to speak a "public number" that ought to be the same if the live clock were interchanged with another live clock; the phase however, is essentially an analog rather than a digital business; the phase is idiosyncratic in the sense that there must be a tolerance within which it would differ in one live clock were substituted for another. Idiosyncratic phases are indispensable to the control that permits the logically coherent transmission of numerals from one live clock to another, so that a "7" on a tape of live clock $A$ becomes a "7" on live clock $B$, and arithmetic functions across a communications channel.

When we set aside the concept of a coordinate system in favor of a network of live clocks, with its feedback loops that act on its patterns of signal exchange in response to unpredictable influences, do we have anything to hold onto in place of the fixed relations that are most vivid in the rigid body that anchors coordinates in special relativity? What a network of live clocks offers is both a communications facility and a conceptual framework to think about connections among entities of interest. This 
conceptual framework enriches the notion of a coordinate system in circumstances in which a coordinate system is appropriate. It also serves in situations in which unpredictability makes a coordinate system unavailable. What one holds onto are the records of the communications channels and of the unpredictable adjustments necessary to their maintenance found in the memories of live clocks.

\section{DISCUSSION}

This paper builds on the more detailed analysis of unpredictability in clocks reported in ref. [3], in which live clocks are called "open machines."

Guesswork as a necessity has a long history of proponents. For example, Einstein endorses Hume's showing that experimental evidence can never establish a causal connection, and Einstein goes further to say that all our concepts depend on "freie Schöpfungen des Denkens"-free creations of thoughti.e. guesses. ${ }^{18}$ The virtue of the proofs noted in Sec. 2 in not to bring a new thought, but to demonstrate it from within physics.

For computation to work over a communications channel, it is necessary for the steps of computation to be adjusted in response to unpredictable, idiosyncratic phases of signal arrivals over logically synchronized channels. Channels in networks get put into operation, are maintained for a while, and then perish. The memory of a live clock can house a picture of the network at some moment, and this picture may or may not agree with a picture held in the memory of another live clock; indeed, communications delays can make two such pictures held in separated memories incommensurate. The coming into operation and the perishing of channels are almost entirely outside the scope of this report: the maintenance of logically synchronized channels alone, while only the simplest part of the story, shows how, by invoking guesses of how to respond to unpredictable measured detections, live clocks step their computations in a way that allows the spread of arithmetic across a network. Attention to the networks of live clocks as references for motion shows ways in which the unpredictable and the calculated work in combination during scientific discovery, and how that "working together" rules out the view that what is discovered is out there independent of we who, with our dependence on guesswork, go about looking for it.

Contact with the physical world is reflected in records held in the memories of computers, seen here as live clocks. Such memories include traditional libraries holding the extant physics literature. The records in memories distributed over networks of computers depend on assumptions, as does the use of these records in making predictions and in designing experiments. The assumptions that reside in memories and are applied, for example in managing logical synchronization, vary in what one might call depth. At root are community-wide assumptions, including conventions of language and logic. From these assumptions, that investigators are slow to change, there range more flexible assumptions, up to those that an individual can make today and drop tomorrow. Under the impact of responses to surprises to which assumptions are vulnerable, this tangle of axioms, postulates, hypothes and whatever else we call it that comes into our heads without a logical justification, which we call a "tree of assumptions," evolves unpredictably; it can be glimpsed by any person only in a small part, and a person's capacity to notice an assumption depends on what other assumptions that person carries or rejects at the moment.

Maintaining logical synchronization among live clocks that contribute to a reference background of motion must draw on a tree of assumptions that puts a gap between evidence and its explanations. 
The numbers that come as evidence into a network affect the network and are distinct from explanations in terms of particles and fields, also numeric structures, which presuppose a coordinate system. Pretending that particles or fields can enter records directly as evidence obscures the gap between evidence and explanation. Accepting the unpredictability in a reference background that stems from the guesswork intrinsic to the tree of assumptions engenders a deeper sense of order, resident in memories of live clocks, and operative in situations beyond those for which the notion of a coordinate system finds application.

Accepting unpredictability in the backgrounds against which all else is seen takes a certain courage. The reward is the opportunity to learn to work with unpredictability in physics, thereby entering a world in which the joy of an answer can be the new question it enables.

\section{Acknowledgment}

We thank Prof. Tai Tsun Wu for discussions of the topic of discovery.

\section{REFERENCES}

1. A. Einstein Relativity: The Special and General Theory (Crown Publishers, New York, 1961) App. V, p. 142.

2. M. Soffel et al., "The IAU resolutions for astrometry, celestial mechanics, and metrology in the relativistic framework: explanatory supplement," The Astronomical Journal, 126, 2687-2706 (2003).

3. J. M. Myers and F. H. Madjid, "Distinguishing between evidence and its explanations in the steering of atomic clocks," Annals of Physics 350, 29-49 (2014); arXiv:1407.8020.

4. The invention of the notion of an oracle is due to A. M. Turing, and can be found in his 1938 thesis, reprinted in Alan Turing's Systems of Logic, the Princeton Thesis, Princeton University Press, Princeton, NJ (2012).

5. J. M. Myers and F. H. Madjid, "A proof that measured data and equations of quantum mechanics can be linked only by guesswork," in S. J. Lomonaco Jr. and H.E. Brandt (Eds.) Quantum Computation and Information, Contemporary Mathematics Series, vol. 305, American Mathematical Society, Providence, 2002, pp. 221-244; arXiv:quant-ph/0003144.

6. F. H. Madjid and J. M. Myers, "Matched detectors as definers of force," Ann. Physics 319 (2005), 251273; arXiv:quant-ph/0404113.

7. J. M. Myers and F. H. Madjid, "What probabilities tell about quantum systems, with application to entropy and entanglement," in A. Bokulich and G. Jaeger, eds., Quantum Information and Entanglement, Cambridge University Press, Cambridge UK, pp. 127-150 (2010); arXiv:1409.5100

8. J. M. Myers and F. H. Madjid, "Ambiguity in quantum-theoretical descriptions of experiments," in K. Mahdavi and D. Koslover, eds., Advances in Quantum Computation, Contemporary Mathematics Series, vol. 482 (American Mathematical Society, Providence, I, 2009), pp. 107-123; arXiv:1409.5678.

9. C. W. Helstrom, Quantum Detection and Estimation Theory, (Academic Press, New York, 1976).

10. A. S. Holevo, Probabilistic and Statistical Aspects of Quantum Theory, (North-Holland Publishing Co., Amsterdam, 1982)

11. A. M. Turing, "On computable numbers with an application to the Entscheidungsproblem," Proc. London Math. Soc., Series 2, 42, 230-265 (1936).

12. C. E. Shannon, "A mathematical theory of communication," The Bell System Technical Journal, Vol. 27, pp. 379-423, 623-656, July, October, 1948. 
13. J. M. Myers and F. H. Madjid, "Rhythms of Memory and Bits on Edge: Symbol Recognition as a Physical Phenomenon," arXiv/1106.1639, 2011.

14. A. Einstein, "Zur Elektrodynamik bewegter Körper," Annalan der Physik, 17, 891-921 (1905).

15. J. F. Sawyer and C. A. Reiter, "Perfect parallelepipeds exist," Mathematics of Computation 80, 1037-1040 (2011) (also arXiv:0907.0220v2).

16. V. Perlick, "On the radar method in general-relativistic spacetimes," in H. Dittus, C. Lämmerzahl, and S. Turyshev, eds., Lasers, Clocks and Drag-Free Control: Expolation of Relativistic Gravity in Space, (Springer, Berlin, 2008); also arXiv:0708.0170v1.

17. The LIGO Scientific Collaboration (http://www.ligo.org) "LIGO: the Laser Interferometer GravitationalWave Observatory," Rep. Prog. Phys. 72, 076901 (2009) doi:10.1088/0034-4885/72/7/076901 http://www.ligo.org

18. A. Einstein, "Bertrand Russell und das philosophische Denken," in Mein Weltbild, (Ullsteinverlag, Berlin, 2005); translation available in Ideas and Opinions, (Crown Publishers, New York, 1954). 